The University of Maine

\title{
DigitalCommons@UMaine
}

Psychology Faculty Scholarship

Psychology

7-1987

\section{Fear Reduction Processes in Imaginal and In Vivo Flooding: A Comment on James ${ }^{\text {Review }}$}

Jeffrey E. Hecker

University of Maine - Main, jeff.hecker@umit.maine.edu

Geoffrey L. Thorpe

University of Maine - Main, geoffrey.thorpe@umit.maine.edu

Follow this and additional works at: https://digitalcommons.library.umaine.edu/psy_facpub

Part of the Psychology Commons

\section{Repository Citation}

Hecker, Jeffrey E. and Thorpe, Geoffrey L., "Fear Reduction Processes in Imaginal and In Vivo Flooding: A Comment on James' Review" (1987). Psychology Faculty Scholarship. 16.

https://digitalcommons.library.umaine.edu/psy_facpub/16 


\section{Fear Reduction Processes in Imaginal and In Vivo Flooding: A Comment on James' Review}

\section{Jeffrey E. Hecker and Geoffrey L. Thorpe \\ University of Maine}

The research comparing imaginal and in vivo exposure in the treatment of clinically significant fear, recently reviewed by James (1986), is reexamined from the perspective of bioinformational theory and the concept of emotional processing. Fear is assumed to be stored in long term memory as a network of propositionally-coded information, which has to be processed if treatment is to be successful. Emotional processing is indicated by activation of fear responses and their habituation within and across treatment sessions. Consistent with the theory, our review indicates that successful treatment via imaginal and in vivo exposure is indeed related to activation and habituation of fear responses; interference with processing has a negative impact upon fear reduction, regardless of the specific treatment techniques employed. Furthermore, some apparently discrepant findings in the available research literature can be understood in terms of the theories cited. These ideas provide a useful perspective from which to plan future research efforts and to advance our understanding of the processes underlying reduction of pathological fear.

\section{Introduction}

In his critical review of the research comparing imaginal and in vivo flooding, James (1986) concludes that the available evidence does not support the widely-held view that in vivo exposure is superior to imaginal flooding in the treatment of clinically significant fear. Although we agree with James' conclusion, and with his warning that it would be premature to abandon imaginal exposure methods, we might wish for some theoretical perspective from which to interpret these conclusions and to make further predictions (see also Barlow and Wolfe, 1981). A suitable perspective is provided by Lang

Reprint requests to Dr. Jeffrey E. Hecker, Department of Psychology, University of Maine, Orono, Maine 04469-0140, U.S.A. 
(1979, 1985), whose bioinformational theory provides a cognitive model of the storage of phobic information in memory, and which postulates possible mechanisms of fear reduction. Foa and Kozak (1986) have expanded upon Lang's basic ideas and have suggested that the concept of emotional processing is helpful in understanding fear reduction. We shall argue that bioinformational theory, coupled with the emotional processing construct, provides a promising vantage point from which to re-examine the literature reviewed by James.

\section{Bioinformational theory}

Lang (1985) proposes that a phobia is represented in long-term memory as a network of propositionally coded information. These propositions can be organized into three categories of information: information about the stimulus characteristics of a feared situation; information about verbal, physiological, and overt behavioral responses; and information about the client's interpretation of the meaning of stimulus and response elements. This information network is considered to be a prototype for emotional expression. Lang has proposed that the network is processed when a critical number of propositions is accessed. Accessing of propositions occurs when there is a match between environmental stimuli and information contained in the phobia network, or when prototypematching information is internally generated (e.g., through imagery). Processing is associated with detectable psychophysiological changes as response propositions are activated (Lang, 1979, 1984).

Following from the theory, subjects who show autonomic changes during imagery are assumed to be processing fear-network information, including the response elements. Several factors influence whether or not changes in heart rate, for example, occur when subjects imagine fearful scenes. First, phobics who rate themselves as good imagers tend to be able to generate fear-relevant physiological changes during imagery (Levin et al., 1982). Second, activation of response elements is facilitated by instructions that explicitly direct subjects to experience fear physiologically. This effect is most pronounced when imagery scripts contain references to muscular and visceral responses (Lang $e t$ al., 1980; Lang et al., 1983). Third, clients' fear classification appears to be related to their ability to process response information during imagery. Simple phobics tend to show the predicted autonomic changes, whereas agoraphobics tend not to do so (McNeil et al., 1983; McNeil et al., 1985).

Fear reduction, according to the bioinformational model, involves breaking down the phobia network. Lang (1984) argues that in order for the phobia network to be dismantled the information must first be processed. 
From the perspective presented here, treatment of phobia would involve a breakdown of network coherence and attachment of the stimulus and meaning information to other response subroutines. Any processing through exposure or imagery would facilitate this goal, as the processing context would necessarily add new, possibly inhibitory or incompatible information to the prototype, reducing coherence through this broader association, and encouraging a division into smaller subprograms that could be instigated independently by differing environmental circumstances. (Lang, 1984, p. 195)

From Lang's viewpoint, then, processing is a necessary condition for the breakdown of the phobic memory structure and hence therapeutic change. Processing of the phobia network would occur relatively consistently when in vivo exposure methods are used, since there would be a near perfect match between the stimulus information available to the client and the stimulus elements of the network (Lang, 1985). Processing of the phobia network is likely to be less consistent when imaginal exposure methods are used since, as we have seen, there are several factors which affect whether or not clients activate response information during imagery. These factors have varied in an uncontrolled fashion between and within studies of imaginal exposure treatments (Lang, 1977).

\section{Emotional processing}

Foa and Kozak (1986) have suggested emotional processing as the mechanism by which the phobic memory structure is modified. Accepting Lang's conception of how phobic information is stored in memory, Foa and Kozak propose that two conditions are required for the reduction of fear. First, as Lang (1977) has suggested, information relevant to the phobia (stimulus, response, and/or meaning) must be made available to a client so that the fear network is activated. Second, additional information must be made available as the phobia network is being processed-information that is incompatible with some of the information in the fear structure-so that a new memory is formed. Emotional processing, then, refers to changes in the fear structure that occur when there is exposure to phobia-relevant information, and information incompatible with the fear network is incorporated into the memory structure.

Foa and Kozak (1986) have examined data from several clinical studies and gleaned a set of three response characteristics that occur with some consistency in clients who improve with exposure treatment. They use this set of responses, therefore, as indicators of emotional processing. The first of these, activation of fear responses, can be inferred when clients show physiological arousal and/or report subjective experiences of fear during exposure. Habitu- 
ation of fear responses is indicated by a gradual decrease in indices of fear within exposure sessions. Across-session habituation is indicated when fear responses to the initial presentation of phobic stimuli decline during subsequent exposure sessions. These effects are associated with successful treatment of phobic and obsessive-compulsive disorders:

The activation of affect, its reduction during exposure sessions, and its decrease across sessions, appear positively related to treatment outcome, denoting evocation and modification of fear memories during therapy (Foa \& Kozak, 1986, p. 23).

\section{Imaginal vs in vivo exposure}

It follows from the points made above that, in order to review the imaginal verses in vivo flooding literature from the emotional processing perspective, certain information must be available to assess whether or not processing has occurred. To allow examination of Foa and Kozak's (1986) three indicators of processing, a report must include the following information. First, in order to assess activation and within-session habituation, information on fear reactions (preferably psychophysiological and self-report) is required. Second, data from multiple treatment sessions need to be examined in order to assess acrosssession habituation. Third, outcome measures that are clearly distinct from the indicators of emotional processing must be available. If the variables used to infer successful treatment are not distinct from those used to infer emotional processing, to conclude that the former is caused by the latter would be tautological.

\section{Activation}

Three of the studies reviewed by James (1986) indicate that activation of fear responses did not occur during imaginal exposure treatment. Watson et al. (1973) used therapists' ratings of anxiety during imaginal exposure sessions to examine arousal levels. The results indicate that their patients were relatively unaroused. Overall mean anxiety rating was 3.06 on a ten point scale. Psychophysiological measures of activation probably provide more useful information since they are free from self-report biases. Stern and Marks (1973) assessed skin conductance and heart rate during "fantasy" flooding. They found "little skin conductance activity or tachycardia" (p. 273) during fantasy sessions. Chambless et al. $(1979,1982)$ similarly found little GSR or heart rate response during pretreatment imagery assessment. This suggests that these subjects were not good imagers and is consistent with other findings with agoraphobic subjects (e.g., McNeil et al. 1985). Recall that imagery instruc- 
tions affect activation of response information during imagery (Lang et al., 1983). It is unclear from the Chambless et al. (1982) report what type of instructions were given to subjects during pre-treatment imagery assessment. The Chambless et al. (1979) study is particularly interesting with respect to the role of activation of fear responses during exposure in fear reduction. This study compared three treatment conditions: imaginal flooding, imaginal flooding with Brevital, and an attention control. Subjects in the two flooding conditions were provided with markedly different imagery instructions. In the imaginal flooding without drugs condition "the therapists urged clients to experience imagined situations as if they were actually occurring, to feel the concomitant anxiety or shame acutely until it finished" (p. 245). In contast, in the Brevital-assisted flooding group, an effort was made to keep clients calm and relaxed throughout imaginal procedures. Brevital is a short-acting barbiturate and likely had its strongest effects on physiological systems, suppressing them during imaginal exposure. The suppressive effects of Brevital along with the relaxing imagery instructions likely served to minimize physiological arousal during imaginal exposure. This has a negative effect on treatment outcome since in this study non-drug imaginal flooding generally led to better outcome than drug-assisted flooding. In addition, no difference in treatment effectiveness were found between standard imaginal flooding (in which clients were urged to experience their anxiety fully) and in vivo exposure (Chambless et al., 1982).

While the Chambless et al. (1979) study suggests that suppression of fear responses during flooding is negatively related to outcome, other researchers have reported the complementary finding that activation of fear reactions appears to be related to positive outcome. For example, Stern and Marks (1973) found that "initial high physiological arousal . . . correlated with a good outcome" (p. 273). Mathews et al. (1976), similarly, found strong fear reactions, both physiological and self-report, to phobic imagery during test probe sessions. This suggests that, unlike clients in other studies, these agoraphobics were able to activate response information during phobic imagery. This is important since in this study no differences in outcome were found between imaginal and real life exposure. This suggests, therefore, that if clients can activate response information during phobic imagery, imaginal exposure treatments may be as effective as in vivo techniques.

\section{Habituation}

The second indicator of emotional processing is habituation of fear reactions within treatment sessions. Three of the studies reviewed by James present data relevant to this issue. In two studies, prolonged exposure sessions proved 
superior to briefer exposure trails (Rabavilas et al., 1976; Stern and Marks, 1973). This result is likely due to the fact that longer sessions allowed for habituation of fear responses within the session. For example, Stern and Marks (1973) found that during long in vivo flooding sessions, heart rate and subjective anxiety decreased more over the second than over the first hour. Similarly, Rabavilas et al. (1976) found that long in vivo exposure sessions led to greater fear reduction than any other treatment. In addition, long imaginal flooding sessions seemed to potentiate the effects of in vivo practice sessions whereas short imaginal flooding sessions did not. This also suggests that allowing fear reactions to phobic imagery to subside is positively related to treatment outcome.

Failure of habituation appears to be related to poor outcome of imaginal exposure. In the Watson et al. (1973) study, therapists' rating of clients' anxiety tended to increase during the course of a single flooding session. While, as discussed previously, these therapists' ratings did not indicate high levels of arousal, arousal levels did not decrease during sessions. Imaginal flooding had been a relatively ineffective intervention in this study.

\section{Across-session habituation}

With the respect to across-session habituation, few studies provide useful information. In some, the research designs and the limited time-span over which the studies were conducted preclude any useful analysis of acrosssessions habituation (Rabavilas et al., 1976; Stern and Marks, 1973; Watson et al., 1973). Two studies provide some data from which across-session habituation can be inferred. Chambless et al. (1982) re-examined the data from their earlier study after additional work with the same clients. In comparing subjects who received standard imaginal flooding to those who received imaginal flooding with Brevital, they concluded that the results favored standard flooding. Examination of the within-session fear ratings provided by subjects during flooding revealed no difference in the pattern of responses within sessions. That is, in both conditions subjects' ratings of anxiety decreased within sessions. However, the conditions did differ in patterns of initial anxiety ratings across sessions. The initial ratings for the non-drug flooding group tended to decrease across sessions. For the drug-flooding condition, initial anxiety ratings remained high across sessions. In other words, in the non-drug condition habituation of anxiety ratings generalized across sessions. No generalization of habituation was found for the drugflooding group. Unfortunately, the number of subjects was too small to apply statistical tests to these observed trends.

Across-session habituation of fear responses was observed for both 
imaginal and in vivo flooding procedures in the Mathews et al. (1976) study. Subjects' heart rates and skin conductance during phobic and neutral imagery were assessed pretreatment, at the midpoint, and post-treatment. In addition, subjective ratings of anxiety were also examined at these points. The same pattern of results was found in all three treatment conditions (imaginal flooding, in vivo exposure, and a combination treatment). The authors report that ". . . on first presentation both physiological (heart rate) and subjective responses to phobic imagery were greater than to neutral imagery, and . . . this disparity was reduced over time as the phobic response declined within and between occasions of testing" (p. 368). While these results ae reported for test probe sessions, not actual treatment sessions, it seems logical that a similar

1 pattern of results would be found for subjects' responses to fearful imagery during imaginal flooding. The fact that the same pattern of habituation of fear responses to phobic imagery occurred regardless of the treatment method employed, suggests that the same mechanism underlies the changes seen in each condition.

\section{* Concluding remarks}

Re-examination of James' (1986) review material has allowed us to evaluate certain predictions drawn from bioinformational theory. Of the six studies cited by James, five provided at least some of the relevant information (the exception being Emmelkamp and Wessels, 1975). Specifically, activation of fear-relevant autonomic responses, and habituation of these responses within treatment sessions, prove to be related to favorable treatment outcome. The hypothesis that across-session habituation would be related to beneficial outcome also receives partial support.

Procedural comparisons of alternative treatments (such as imaginal and in vivo exposure) are undoubtedly of practical importance. Yet theory-based research is equally important, since it allows elucidation of crucial treatment processes, in addition to its heuristic value in guiding our investigations. Fortunately, these two approaches are not incompatible. As we have tried to show, the addition of theoretical questions from bioinformational theory to empirical questions about treatment effectiveness has the potential to help resolve apparent conflicts in the results of reported studies. This approach has helped to show that it is the degree of processing encouraged by a procedure, rather than an arbitrarily-labeled treatment technique itself, that is correlated with outcome in the treatment of phobic anxiety. To illustrate the heuristic value of this approach, we can cite an implication of Lang's theory, currently under investigation in our research clinic, that prior training in activation of response information will potentiate the effects of exposure, imaginal or real-life, to fear-relevant stimuli. 


\section{References}

BARLOW, D. H. and WOLFE, B. (1981). Behavioural approaches to anxiety disorders: A report on the NIMH-SUNY research conference. Journal of Consulting and Clinical Psychology 49, 448-454.

Chambless, D. L., FoA, E. B., Groves, G. A. and Goldstein, A. J. (1979). Flodding with Brevital in the treatment of agoraphobia: Countereffective? Bebaviour Research and Therapy 17, 243-251.

Chambless, D. L., FoA, E. B., Groves, G. A. and Goldstein, A. J. (1982). Exposure and communication training in the treatment of agoraphobia. Bebaviour Research and Therapy 20, 319-328.

EMmelkamp, P. M. G. and Wessels, H. (1975). Flooding in imagination versus flooding in vivo: A comparison with agoraphobic. Bebavior Research and Therapy 13 , $7-15$.

FOA, E. B. and KOZAK, M. J. Emotional processing of fear: Exposure to corrective information. Psychological Bulletin 99, 20-35.

JAMES, J. E. (1986). Review of the relative efficacy of imaginal and in vivo flooding in the treatment of clinical fear. Bebavioural Psychotherapy 14, 183-191.

LANG, P. J. (1977). Imagery in therapy: An information processing analysis of fear. Behavior Therapy 8, 862-868.

LANG, P. J. (1979). A bioinformational theory of emotional imagery. Psychophysiology $16(6), 495-512$.

LANG, P. J. (1984). Cognition in emotion: Concept and action. In Emotion, Cognition, and Bebavior C. Izard, J. Kagan and R. Zajonc (Eds), pp. 192-225. New York: Cambridge University Press.

LANG, P. J. (1985). The cognitive psychophysiology of emotion: Fear; and anxiety. In Anxiety and the Anxiety Disorders, A. H. Tuma and J. D. Maser (Eds), pp. 131-170. Hillsdale, N.J.: Lawrence E. Erlbaum Associates.

Lang, P. J., Kozak, M. J., Miller, G. A., Levin, D. N. and Mclean, A. (1980). Emotional imagery: Conceptual structure and patterns of somato-visceral response. Psychaphysiology 17, 179-192.

LANG, P. J., Levin, D. N. , Miller, G. A. and KozaK, M. J. (1983). Fear behavior, fear imagery, and the psychophysiology of emotion: The problem of affective response integration. Journal of Abnormal Psychology 92, 276-306.

Levin, D. N. , CooK, E. W. and LANG, P. J. (1982). Fear imagery and fear behavior: Psychophysiological analysis of clients receiving treatment for anxiety disorders. Psychopbysiology 19, 571-572 (abstract).

Mathews, A. M., Johnston, D. W., Lancashire, M., Munby, M., Shaw, P. M. and Gelder, M. G. (1976). Imaginal flooding and exposure to real phobic situations: Treatment outcome with agoraphobic patients. British Journal of Psychiatry 129, 362-371.

McNeil, D. W., Melamed, B. G., Cuthbert, B. N. and Lang, P. J. (1983). Emotional imagery and psychophysiological responsivity in simple phobic and agoraphobia. Psychophysiology 20, 459 (abstract). 
MCNeIL, D. W., VRana, S. R., Melamed, B. G. and Lang, P. J. (1985). Affective response to fear imagery: Individual differences in phobic populations. Poster presented at the meeting of the Association for the Advancement of Behavior Therapy. Houston, Texas, November.

rabavilas, A. D., Boulougouris, J. C. and Stefanis, C. (1976). Duration of flooding sessions in the treatment of obsessive-compulsive patients. Behaviour Research and Therapy 14, 349-355.

STERN, R. and MARKS, I. (1973). Brief and prolonged flooding: A comparison in agoraphobic patients. Archives of General Psychiatry 28, 270-276.

W ATson, J. P., Mullett, G. E. and Pillay, H. (1973). The effects of prolonged exposure to phobic situations upon agoraphobic patients treated in groups. Bebaviour Research and Theratpy 11,. 531-545.

(Date received: November 1986) 